\title{
TURISMO GASTRONÓMICO Y VINO: ANÁLISIS DE LA OFERTA GASTRONÓMICA Y HOSPEDAJE EN MONTILLA Y MORILES
}

\author{
PROF. DR. RICARDO DAVID HERNÁNDEZ ROJAS ${ }^{1}$ \\ UNIVERSIDAD DE CÓRDOBA \\ PROF. JOSE ANTONIO CAVA JIMÉNEZ ${ }^{2}$ \\ UNIVERSIDAD DE CÓRDOBA
}

\section{Resumen}

El turismo es uno de las acciones más enriquecedoras, motivadoras y positivas que realiza el ser humano, y aún más si se enlaza con la gastronomía y el vino. El turismo gastronómico ofrece productos autóctonos, cocina del lugar, hospitalidad, costumbres, festivales, etc., Así pues, el turismo gastronómico se ha convertido en un turismo emergente pero ya consolidado, que satisface en un mayor grado las necesidades que se le plantean hoy en día al consumidor turístico. Esta nueva modalidad de turismo aparece como alternativa al que tradicionalmente se realizaba. Es una de las más demandadas por la sociedad, dando lugar a una gran oportunidad de crecimiento económico en las zonas rurales que gozan de producción vinícola y rica gastronomía. Las Denominaciones de Origen, las bodegas, las materias primas autóctonas forman parte del territorio de una región y conforman un atractivo para el turista. El desarrollo de rutas de vino, actividades implícitas como catas, gastronomía, estancias y experiencias turísticas relacionadas con estas visitas proporciona una fuente de ingresos adicional para el destino, a la vez que suponen una mayor difusión de los municipios y de los territorios. El objetivo de este trabajo se basa en el análisis de la oferta tanto de restauración como de hospedaje existente a lo largo de los municipios de Montilla y Moriles.

Del análisis realizado se desprende que el desarrollo del turismo basado en la visita a Montilla y Moriles, vía denominaciones de origen, ruta del vino dependerá en buena medida de la interrelación de los servicios de restauración y hospedaje, las administraciones públicas y entes locales y la comercialización en general de la visita establecida, siendo estos factores tras el análisis realizado, reflejo del alto grado potencial y crecimiento turístico que tiene la región.

\footnotetext{
${ }^{1}$ Profesor del departamento de Economía, Sociología y Política Agrarias. Universidad de Córdoba, España, et2heror@uco.es

${ }^{2}$ Profesor Asociado del departamento de economía, sociología y política Agrarias, Universidad de Córdoba, España. jcava@uco.es
} 
Palabras clave. Turismo gastronómico, Denominación de Origen, hospedaje.

\section{Abstract}

Tourism is one of the most enriching and positive actions performed by the human being, and even more if it is linked with gastronomy, wine and rural areas. Gastronomic tourism offers local products, local cuisine, hospitality, customs, festivals, etc. Thus, gastronomic tourism has become an emergent tourism but already consolidated, which satisfies to a greater degree the needs that arise today In day to the tourist consumer. This new form of tourism appears as an alternative to what was traditionally carried out, is currently one of the most demanded by society, giving rise to a great opportunity for economic growth in rural areas that enjoy wine production and rich gastronomy. Denominations of Origin, wineries, native materials are part of the territory of a region and are an attraction for the tourist. The development of routes, implicit activities, gastronomy, stays and tourist experiences related to these visits provides an additional source of income for the destination, while implying a greater diffusion of municipalities and territories. The objective of this work is based on the analysis of the offer of both restoration and lodging existing throughout the municipalities of Montilla and Moriles.

From the analysis made, it follows that the development of tourism based on the visit to Montilla and Moriles, via appellations of origin, own wine route will depend to a great extent on the interrelation of catering services and public administrations and local and The marketing in general of the established visit, being these factors after the analysis realized, reflecting the high potential degree and tourist growth that the zone has.

Key words. Gastronomic Tourism, Protected Designation of Origin, accomodation.

\section{Introducción}

El sector turístico tiene una importancia clave en la economía española tanto desde el punto de vista cuantitativo como desde una perspectiva cualitativa (Morillas, 2001), es clara la importancia que representa la actividad turística en el producto interior bruto representa más de un $11 \%$ del mismo y un 13\% del total del empleo en el año 2015. Desde una óptica cualitativa, la innovación tecnológica ha introducido cambios relevantes en el comportamiento del sector al permitir una más rápida transmisión de la información y una reducción de los costes de transporte, unidos estos al desarrollo de la actividad turística.

El sector turístico en Andalucía ha alcanzado una relevancia económica, que ha permitido mejorar los desequilibrios económicos y compensar la balanza de pagos generados por la crisis económica, el peso que representa el producto interior bruto generado por el turismo alcanza en esta comunidad el $12,5 \%$, un punto y medio por encima de la media nacional. Córdoba y provincia presentan igualmente cifras similares pero teniendo un peso del sector servicios dentro de la economía superior al 67\%, esto nos lleva a concluir que como destino turístico de interior cuenta con una gran potencialidad tanto cualitativa como cuantitativa (Hernández et al, 2016)

El patrimonio cultural y la gastronomía son una de las motivaciones principales en la elección de destino turístico (Muñoz- Fernández et al, 2016). El turista de esta década no solo tiene una motivación exclusiva para el viaje sino varias: gastronómico, 
tipo de hospedaje, enológico, patrimonial etc; entre las características principales del turista gastronómico podemos citar cuatro: poder adquisitivo medio-alto, viaja acompañado, aprecia la calidad junto a la singularidad y por último exige profesionalidad (Hernández et al, 2016)

Las bodegas de vino enlazadas empresarial y turísticamente en forma de ruta gastronómica presentan características propias que los convierten en verdaderos recursos turísticos, es decir, su singularidad es la atrayente para el turista. Diferentes autores desde hace más de dos décadas han destacado como una de las motivaciones en el turismo es la búsqueda de la autenticidad y han formulado investigaciones en relación a la misma (MacCannel,1976) (Gobbi, 2003), siguiendo a estos autores, han surgido teorías sobre la difícil interpretación que manifiestan dar autenticidad a determinados viajes: sol y playa, viajes a Parques Temáticos de atracciones (Shudson,1979;Stephen, 1990;Urry,1990). Se entiende pues que cada zona geográfica natural debe buscar su propia identidad para acercarse a la autenticidad. La oferta gastronómica y de hospedaje es un factor esencial el cual puede poner lo original en valor (Hernández, 2016)

En Córdoba y provincia existen definidos los distintos lugares o enclaves que pueden ser objeto de esta tipología de turismo: los municipios de Montilla y Moriles son el origen de la Denominación de Origen que lleva su nombre. Esta investigación pretende tener un conocimiento más exhaustivo y preciso de toda la oferta en hostelería y de hospedaje referidas a esta tipología de Turismo gastronómico que se pueden dar en la zona de Montilla-Moriles, epicentro de la Denominación de Origen protegida que lleva su nombre, siendo esto novedad que presenta este artículo.

La consecuencia al objetivo específico de este trabajo es la catalogación y clasificación de la oferta gastronómica y hospedaje de los dos municipios que dan nombre a la ruta del vino en Córdoba: Montilla y Moriles detectando fuentes de oportunidad para el incremento del turismo en la zona.

El documento consta de cuatro partes. En primer lugar, se define el marco teórico del turismo gastronómico relacionado con el vino, a continuación se muestra la ubicación de la zona objeto el estudio, en tercer lugar se desarrolla la metodología usada para terminar con los resultados obtenidos del trabajo de campo realizado y las conclusiones obtenidas del mismo.

\section{Marco teórico en materia de turismo gastronómico del vino}

Las diferentes investigaciones académicas en cuanto al turismo del vino se inicia en la década de los 90, centrado en el análisis del impacto socioeconómico y en la conducta del turista que visita las bodegas, elementos que investigaron en un principio (Hall, 1996) y seguidamente autores como Sharple, Cambourne, Macionis (Hall et al ,2000).

El enoturismo ha experimentado un desarrollo importante en todo el mundo. Australia, Nueva Zelanda, California, Sudáfrica y América del Sur son las nuevas fronteras del enoturismo y en consonancia los escritos científicos se han ido desarrollando en estas nacionalidades. Los investigadores que comparten la idea de que el enoturismo puede ser generador de riqueza en las zonas de interior y su importancia estratégica, podemos sintetizarlos por paises: Australia (Dowling y Carlsen, 1999), Canadá ( Telfer, 2001; Williams y Nelly, 2001; Willlians y Dossa, 2003; Hashimoto y Telfer, 2003), Chile (Sharpes, 2002), Francia (Thevenin, 1996; Frochot, 200), Hungría (Szivas, 1999), Italia, Nueva Zelanda (Beverland, 1998; Mitchell y Hall, 2003), 
Sudáfrica (Preston-Whyte, 2000; Bruwer, 2003; Demhardt, 2003), los EEUU (Skinner, 2000), Reino Unido (Howley \& Van Westering, 2000) y España (Millán, Guzmán y Agudo, 2006; Melián, Millan y López-Guzmán, 2008;Hernández,2015;Millan et al,2016).

Esta bibliografía indica que el turismo gastronómico ha dejado de ser, por tanto, un producto turístico localizado en determinados países con tradición culinaria y vinícola, como puede ser el caso de Francia, para pasar a ser un turismo universal y que puede ser realizado en cualquier región o comarca que cuente con un producto de calidad. Así, Domingos y Henriques (2015) corroboran esta afirmación con el estudio sobre los dulces tradicionales del Algarve como elemento relevante de producto turístico regional, que actúa de dinamizador de la gastronomía y turismo de dicha zona.

Si se formulan productos turísticos adecuados, el alimento y el vino no sólo acrecienta el valor de un destino sino también el propio; En el territorio y el alimento subyace la identidad local (Barrera, E. y Bringas, O.; 2008). La gastronomía, como recurso turístico, no sólo es apreciada por su propio valor intrínseco sino también por su carácter simbólico, en la medida en que actúa como identificador de pueblos y territorios (Armesto, X. A. y Gómez, B; 2004).

En cuanto a autores que presentan investigaciones con respecto a los municipios de Montilla y Moriles podemos destacar aquellos que han escrito sobre la unión de las denominaciones de origen de vino respecto al turismo en la zona, los más numerosos (Millán, 2008),(Guzman et al,2008), (Millán et al, 2010),(Morales, 2010),(Millan,2011),(Millán, 2012),(Millán, 2015), (López-Guzman et al, 2016), con respecto a la valoración económica de las denominaciones de origen y su relación con rutas gastronómicas (Hernández,2015) su impulso económico y comercial vía plan estratégico (Fuentes, 2000) o desde el punto de vista comercial englobado en las cooperativas de la zona (Gómez, 1990) o con respecto al clima de la zona y su repercusión en el vino (Castillejo,1994), desde la perspectiva de la propia uva (Medina, 1988) y ) para concluir incluso con respecto al a la internacionalización y exportación de los vinos (Luque-Vilchez,2015). En cuanto al análisis de la oferta en restauración y hospedaje en la zona de Montilla-Moriles y la Denominación de Origen ningún autor ha realizado dicho estudio, siendo novedoso en el mismo.

\section{Ubicación geográfica de Montilla y Moriles, la denominación geográfica protegida y la ruta del vino}

Córdoba y su provincia se sitúa en la región sur de España y pertenece a la comunidad autónoma de Andalucía, cuenta con una ubicación privilegiada en cuanto a comunicaciones con las principales urbes españolas tanto por AVE (conexión con Madrid, Barcelona, Valencia, Sevilla, Málaga...) como por carretera (Figura 1). En los últimos años como consecuencia del motor económico que es el turismo, se ha empezado a consolidar el crecimiento de la demanda experimentado en la capital en los últimos años dando como resultados atraer a nuevos turistas, diversificar los mercados emisores, mejorar la competitividad de las empresas turísticas o incrementar la rentabilidad de la actividad turística en la ciudad; (Escuela de Organización Industrial, 2014) estas metas se deben de aplicar igualmente a la provincia de Córdoba y en este caso vía a los profesionales dedicados al turismo, administraciones locales y nacionales que operan en torno a la economía de la zona de Montilla Moriles 
Figura 1: Situación de Montilla-Moriles en España.

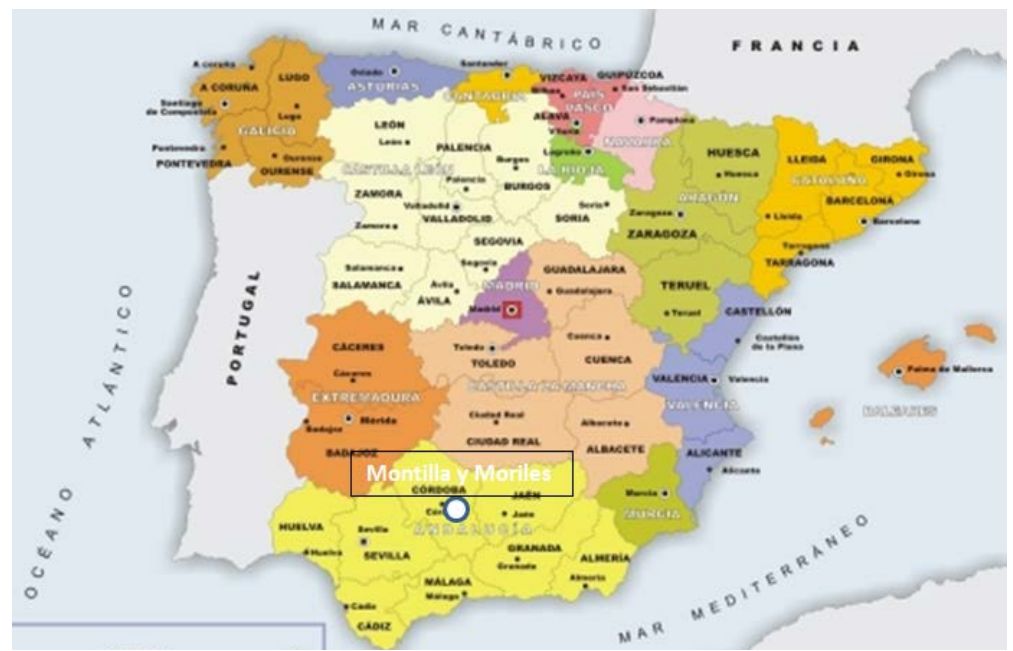

Fuente: elaboración propia a partir de google maps (2017)

Los municipios de Montilla y Moriles, objeto de esta investigación, se encuentran a 46 y 52 kilómetros respectivamente por carretera desde Córdoba capital (Figura 2). La riqueza industrial de Montilla y Moriles y por ende de la zona es la industria del vino. Estudiar la zona de Montilla y Moriles es hablar de la vitinicultura (Titos, 1990). La vid en producción cuenta con más del 80 \% pertenecientes a la zona de Montilla Moriles junto a más del 95\% de las empresas productoras en relación con el vino.

Figura 2: Situación de Montilla y Moriles con respecto a la capital de la provincia.

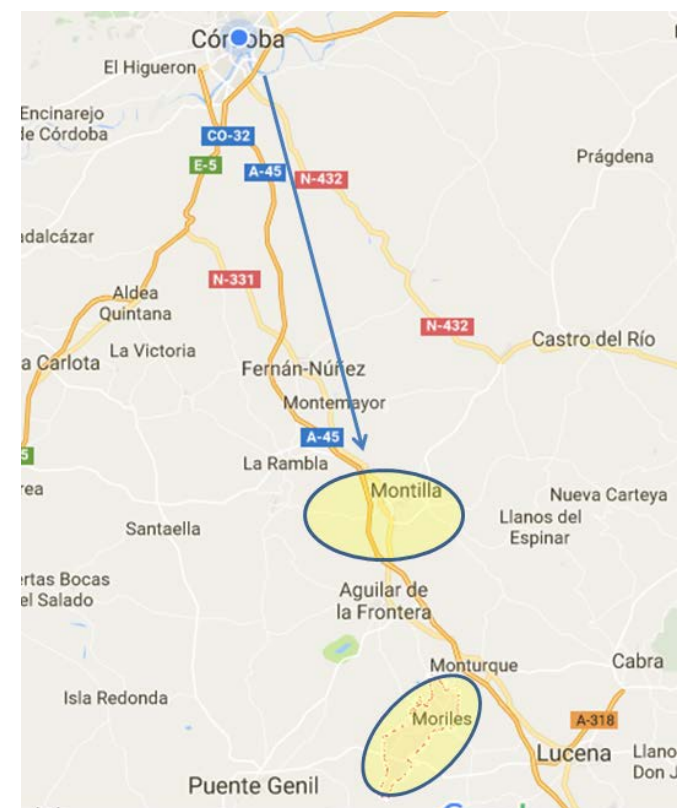

Fuente: elaboración propia a partir de google maps (2017) 
Con respecto al marco de producción, las características climáticas, socioeconómicas y sus implicaciones con el turismo en la zona, desde el punto de vista del turismo no se entienden los municipios de Montilla y el de Moriles aislados sino englobados dentro de la Denominación de Origen y la ruta que da origen.

El marco de producción de la Denominación de Origen se extiende por buena parte del sur de la provincia de Córdoba. Los ríos Genil y Guadajoz limitan sus fronteras oriental y occidental, en tanto que el Guadalquivir, al Norte, y las Sierras de la Subbética, al sur, delimitan su contorno. Formado los términos municipales de Montilla, Moriles, Doña Mencía, Montalbán, Monturque, Nueva Carteya y Puente Genil en su totalidad, y en parte los de Aguilar de la Frontera, Baena, Cabra, Castro del Río, Espejo, Fernán-Núñez, La Rambla, Lucena, Montemayor y Santaella. Figura 3.

\section{Figura 3: Localización de la Denominación de Origen Protegida Montilla Moriles en Córdoba}

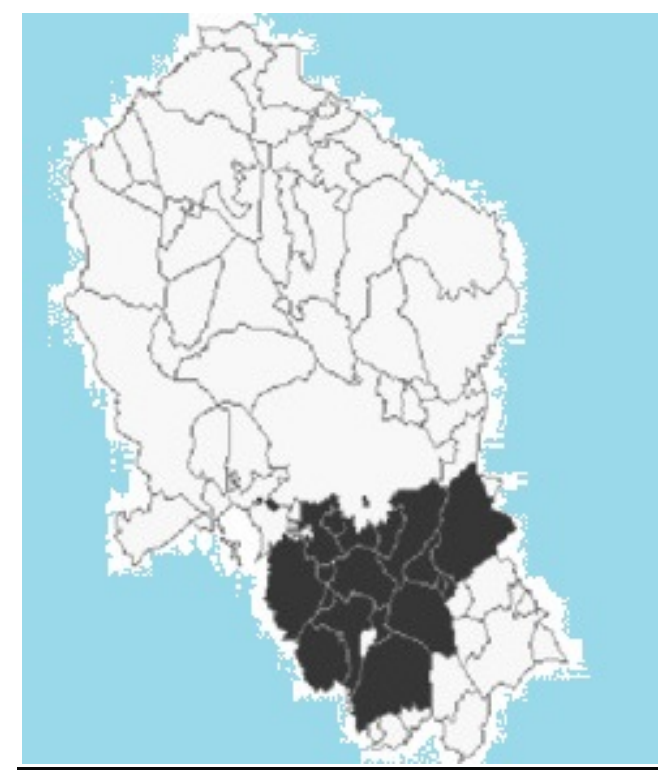

Fuente: Elaboración propia

El clima de la Denominación Montilla-Moriles es básicamente mediterráneo pero con cierto carácter continental con influencia de los vientos que, proceden del Atlántico y entran por el Valle del Guadalquivir. Los inviernos son fríos y los veranos calurosos, largos y secos con una temperatura media máxima superior a $30^{\circ} \mathrm{C}$ y su pluviometría es escasa. Estas condiciones climáticas hacen que sea la primera vendimia de España, y que la obtención de los vinos sea totalmente natural.

Esta zona se encuentra relativamente bien comunicada, dado que desde Córdoba capital la principal vía de comunicación es la autovía, no obstante con las diferentes capitales de provincia de su entorno como son: Sevilla, Granada y Málaga tiene conexión directa por autovía. Asimismo, también está cerca de dos aeropuertos internacionales (Sevilla- Málaga), lo cual es un elemento decisivo para que ciudadanos de otros países consuman este producto turístico. 
Dentro de la D.O. se distinguen dos zonas: una Zona de Calidad Superior localizada en la Sierra de Montilla y los Moriles Altos, donde la tierra albariza provee las mejores condiciones para la obtención de vinos de calidad y el resto otra denominada Zona de Producción.

Con respecto a los datos de población de la zona según los datos del IEA del 2015 (hace referencia a la población de los municipios que forman parte en su totalidad a la D.O.) es de 74.412 habitantes en comparación a los 74.653 habitantes existentes en 2008 destacando el crecimiento negativo poblacional. El municipio de Puente Genil es el municipio que aporta mayor número de habitantes (30.167 habitantes) seguido de Montilla (23.519 habitantes) estos dos municipios concentran la mayor parte de la población. El resto de municipios de la zona se poseen una población entre $1.000 \mathrm{y}$ 5.000 habitantes. Los habitantes mayores de 50 años suponen el 36,88\% de la población de la zona de la D.O. y el 15,39\% de la población son menores de 15 años.

Según los datos observados de SIMA, el número de trabajadores eventuales agrarios subsidiados es de 4004 personas, el 70\% de ellos son mujeres. Además, el paro de este colectivo representa aproximadamente el 55\% de la población parada. Las mujeres podrían ser uno de los colectivos más beneficiados si el turismo se desarrollase en la zona. Pues el turismo gastronómico genera emplea que se adapta más al perfil de estos colectivos (Millan,2008).

La actividad económica generada a partir del sector primario tiene una especial importancia para los municipios que forman la D.O. Montilla-Moriles. En los últimos años esta actividad está impulsando un emergente y cualificado sector industrial auxiliar y de transformación agroalimentaria, como puede ser el vino, el aceite, la aceituna de mesa, etc. Igualmente existe un gran potencial trasformador alrededor de la industria del ajo, Montalbán es unos de los principales productores de este producto a nivel nacional.

En cuanto al turismo existe un escaso desarrollo con respecto a otras rutas del vino, aunque en los tres últimos años ha sufrido un crecimiento aunque no ha superado al año 2011. Entre sus hitos está el conseguir en el año 2005 la certificación como Ruta del Vino de España consiguiendo ser de esta forma la primera ruta certificada de Andalucía.

El producto turístico "Ruta del Vino Montilla-Moriles" consiste en la integración de los recursos y servicios turísticos de interés de una zona vitivinícola, con el fin de construir un producto y/o destino turístico a partir de la calidad e identidad del destino, optimizando la comercialización conjunta, el nivel de satisfacción de la demanda, y el desarrollo socio-económico integral del territorio.

La Ruta actualmente transcurre por el sur de la provincia de Córdoba, en la campiña, y atraviesa 14 municipios incluida la capital de la provincia: Córdoba, Aguilar de la Frontera, Fernán Núñez, La Rambla, Lucena, Montemayor, Montilla, Moriles, Montalbán, Monturque, Castro del Río, Doña Mencía, Santaella y Puente Genil. Esta zona conserva el encanto de un paisaje dominado por viñedos y olivos, una biodiversidad difícilmente alcanzable en otras zonas vitícolas y acompañado de una gran riqueza Histórica, Artística y Patrimonial de los pueblos que la conforman objeto de futuras investigaciones.

Actualmente, según los estudios del observatorio de las rutas del vino de España, la ruta que más visitantes ha tenido ha sido la Ruta del Vino y el Cava del Penedés que engloba a dos de las D.O.P la de Cava y Penedés, siendo la D.O.P Montilla-Moriles con 
19627 visitas en el ranking la número 20 de las 24 rutas que conforman a nivel nacional la asociación española de rutas del vino, ACEVIN estos datos los podemos observar en el gráfico 1.

Gráfico 1: Datos de visitantes en bodegas y museos de las rutas del vino.2015.

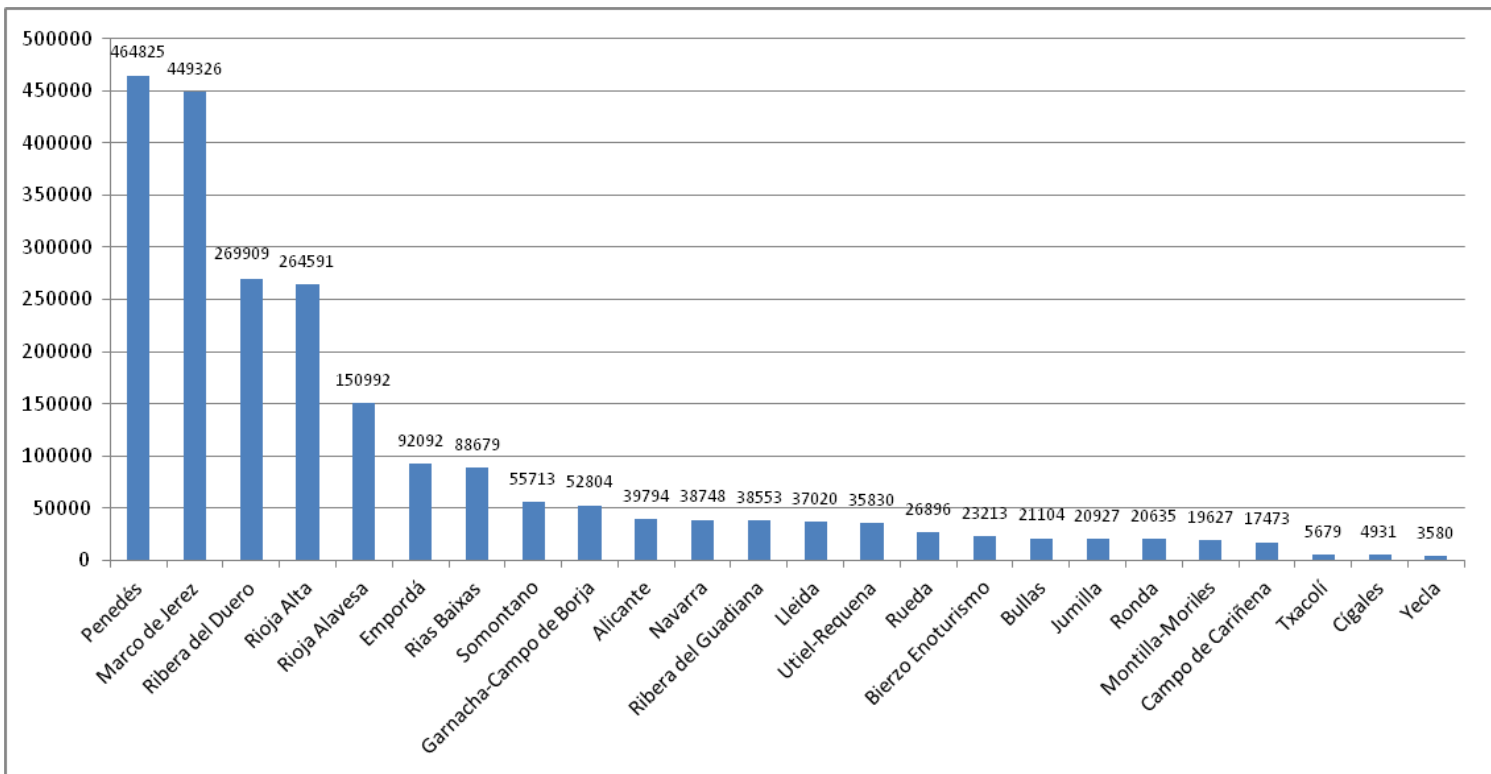

Fuente: Elaboración propia a partir de ACEVIN- Rutas del Vino de España

Así mismo si observamos la evolución del número de visitantes a la ruta Montilla-Moriles a lo largo de los últimos 6 años y tomando de base el año 2011, con 19785 turistas la cifra ha decrementado en el año 2015 en 185 turistas, observamos así mismo como no hay una evolución positiva sino altibajos en la evolución de visitas, estando en el año 2015 por debajo de niveles del año 2011.

\section{Gráfico 2: Evolución del número visitantes a la ruta Montilla-Moriles}

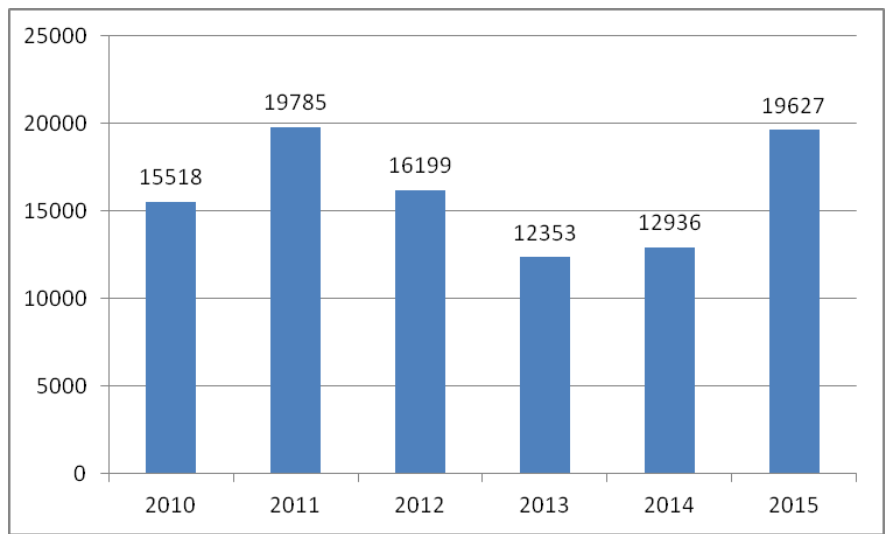

Fuente: Elaboración propia a partir de ACEVIN- Rutas del Vino de España 
En conclusión, podemos decir que aunque la Denominación de Origen Montilla-Moriles la componen 7 municipios en su totalidad y la ruta del vino MontillaMoriles lo integran un total de 14 municipios siendo el epicentro los dos municipios que dan nombre tanto a la ruta como a la Denominación de Origen Protegida existe una evolución desfavorable del turismo, según datos de estudios del observatorio de las rutas del vino de España. Aún estamos lejos de ser estar en los primeros puestos en cuanto a número de turistas junto a la evolución en el tiempo del número de turistas no se observa un crecimiento en los últimos 6 años.

\section{Metodología}

Con el fin de conseguir analizar el objetivo propuesto en la investigación se ha realizado un análisis de alojamiento y restauración en los municipios de Montilla y Moriles, se confecciona una tabla donde se muestra el número de dichos establecimientos junto a datos de carácter cualitativo: tipo de cocina. La Denominación de Origen Protegida comprende estos dos municipios que dan sentido a su nombre: Montilla, aporta el 60,4\% de las bodegas, y Moriles que aporta el 39,6 \% restante. Estos municipios están separados por 23 kilómetros por carretera, figura 2. Se entiende pues que dada la cercanía entre ambas localidades y a su vez compartir territorialmente la Denominación de Origen Protegida el análisis de alojamientos y restauración se circunscribe a los propios municipios y al territorio entre ambos.

El método del estudio de caso tiene principalmente dos funciones en la construcción de teorías (Eisenhardt,1989). La primera consiste en comprobar la congruencia con hipótesis a priori que se han derivado de la teoría existente (en particular, para determinar si el estudio de caso refuta la teoría existente o se presenta como una anomalía).

Los estudios y comparaciones de casos también son valiosos para generar nuevas hipótesis o modelos. Se trata de la construcción de teoría inductiva, y siempre existe la posibilidad de aprender algo completamente nuevo, especialmente en campos como el turismo gastronómico, religioso o el oscuro donde la investigación teórica no es muy abundante. Estudios comparativos de casos que se llevan a cabo con posterioridad podrán determinar si los resultados de los primeros estudios (que desarrollaron un prototipo) son generalizables.

La metodología utilizada para esta investigación, ha consistido:

1. Búsqueda de información en anuarios del Ministerio de Agricultura Alimentación y Medio Ambiente (MAGRAMA) de España, del Instituto de Estadística y Cartografía de Andalucía (datos de ambos municipios y datos sobre la Denominación de Origen Protegida), así mismo en la página web de la ruta del vino y una búsqueda vía internet revisando los 20 primeros ítems encontrados vía buscador google, en paralelo se han consultado las web oficiales de los ayuntamientos para conocer los sitios o lugares de alojamiento y hostelería, así como en la web de la Junta de Andalucía y la propia de la denominación de origen Montilla-Moriles.

2. Entrevistas a profesionales que realizan rutas de turismo enológico tanto en la provincia como en la capital, con el objetivo de conocer sus motivaciones y valoraciones respecto a la potencialidad de ambos municipios. 


\section{Resultados}

Los resultados obtenidos en cuanto a tipo de alojamiento y el número de plazas se muestran en la tabla número 1 . En la oferta de alojamiento vemos como no existen todas las tipologías. No existen ni pensiones ni hostales denotando una falta de variedad en cuanto a esta tipología. Esto significa perdida de potenciales turistas que quieren ese tipo de alojamiento. Esto no es un dato positivo, dado que no se apertura los alojamientos a diferentes tipos de clientes. El hotel de máxima categoría es de tres estrellas, ubicado este en el municipio de Montilla, puesto que en el Municipio de Moriles solo existe un establecimiento de tipología casa rural.

El número total de alojamientos en ambos municipios es 9, de dichos alojamientos 8 en concreto si contemplan el servicio de restauración, dado que los clientes les solicitan estos servicios gastronómicos, siendo sintomáticos tanto de la riqueza gastronómica de la zona como de la tipología de cliente que llega. El 44,4\% de los establecimientos son de la tipología de casa rural, esto es lógico dado la zona de estudio que estamos realizando, aunque si debemos destacar como el número de plazas que aportan al total de la oferta es de un $20 \%$. El número escaso de plazas confrontado con el número de visitas a la Denominación de Origen, 19627 en el año 2015, nos lleva a determinar que no se ofrecen alternativas para que la ruta dure más de un día generando de esta forma pernoctaciones, dando lugar a una escasa oferta en alojamiento. En cuanto al número de estrellas de los hoteles la máxima categoría se ofrece de tres estrellas. Como hemos comentado anteriormente el turista gastronómico y enológico se caracteriza por solicitar servicios profesionales y en consecuencia alojamientos de alta categoría en estrellas, algo de lo que carece la zona de estudio.

Tabla 1: número de alojamientos por tipología.

\begin{tabular}{|l|c|c|c|c|}
\hline \multicolumn{1}{|c|}{$\begin{array}{c}\text { Tipo de } \\
\text { alojamiento }\end{array}$} & \multicolumn{2}{|c|}{$\begin{array}{c}\text { Número de } \\
\text { Establecimientos }\end{array}$} & Número de plazas & $\begin{array}{c}\text { Incluye } \\
\text { Restauración }\end{array}$ \\
\cline { 2 - 5 } & Montilla & Moriles & & \\
\hline Casa Rural & 3 & 1 & 56 & 4 \\
\hline Pensión & \multicolumn{2}{|c|}{0} & 0 & 0 \\
\hline Hostal & \multicolumn{2}{|c|}{0} & 0 & 0 \\
\hline Hotel & 5 & 0 & 224 & 4 \\
\hline Total & & $\mathbf{9}$ & $\mathbf{2 8 0}$ & $\mathbf{8}$ \\
\hline
\end{tabular}

Fuente: elaboración propia

Los resultados obtenidos en cuanto al número de establecimientos se muestran en la tabla número 2, siendo un total de 38 establecimientos, en cuanto a la gastronomía detectada 10 de ellos, un 26,31 \%, presentan un formato con cocina denominada "tradicional del municipio" esto es aquella Cocina que usa como materia prima principal en los platos productos autóctonos de la zona o región así como la oferta 
gastronómica son platos tradicionales de la zona tanto en elaboración, ejecución como en presentación (Romero,2008)

Tabla 2: número de alojamientos por tipología.

\begin{tabular}{|l|c|c|}
\hline \multirow{2}{*}{ Tipo de gastronomía } & \multicolumn{2}{c|}{$\mathbf{N}^{\mathbf{0}}$. Establecimientos } \\
\cline { 2 - 3 } & Montilla & Moriles \\
\hline Bares, Cafeterías & 13 & 11 \\
\hline Tradicional del municipio & 10 & 0 \\
\hline Temáticos (Italiano, Kebab) & 1 & 3 \\
\hline Total por municipio & $\mathbf{2 4}$ & $\mathbf{1 4}$ \\
\hline Total & \multicolumn{2}{|c|}{$\mathbf{3 8}$} \\
\hline
\end{tabular}

Fuente: elaboración propia

En cuanto a la tipología de restaurantes en Montilla predomina por número el tipo "Bar-cafetería" siendo este grupo muy heterogéneo, que unifica cafés, bares, discotecas, bocadillerías, etc. (Romero,2008) además de establecimientos que suministra helados, batidos, refrescos y bebidas en general, sirve al público, a cualquier hora, dentro de las que permanezca abierto el establecimiento, platos fríos o calientes, simples o combinados. En cualquier caso la tipología "restauración tradicional del municipio” tiene una representación importante.

En cuanto al municipio de Moriles la oferta gastronómica si está más enfocada hacía bares y cafeterías, incluso aparecen tres establecimientos temáticos de otras nacionalidades: Italia y Arabia.

La zona de Montilla y Moriles tienen una riqueza y singular gastronomía. La elaboración de platos y postres con vinos de la zona prueba esa singularidad y esa unión culinaria. El maridaje de los platos con los vinos es otra vía de interés gastronómico de la zona (Millán, 2008)

Las elaboraciones de platos Montillanos singulares de la zona destacamos los "riñones al Montilla", apoyados los vinos finos. "Alcachofas a la montillana" preparadas con vino amontillado, "solomillo al oloroso" y la "merluza al Pedro Ximénez con pasas”, y entre los más simples pero no por ello menos complejos en sabor el "picadillo de tomate o naranja" y "el gazpacho". Con el mosto del vino se elaboran platos como "gachas de mosto", y el "arrope", líquido denso, oscuro y muy dulce, que se utiliza para la elaboración de otros platos. Entre las especialidades confiteras montillanas están los "alfajores”, "rosquitos de vino”, "pastelón de cabello de ángel” que mantienen una elaboración artesanal ancestral dando esa singularidad que todo turista gastronómico y enológico busca.

Por otro lado, en Montilla, encontramos la gastronomía vinculada a ciertas fiestas, como son las "gachas con cuscurros" en el día de Todos los Santos, o "pestiños y borrachuelos” en Semana Santa. Estas fiestas atraen a turistas de la zona y a 
poblaciones nacidas en la zona pero que tienen su residencia fuera de municipio. En estos periodos festivos son celebraciones potencialmente atrayentes para el turista.

Ejemplos de eventos consolidados y donde se muestra la rica gastronomía unida al vino es la cata anual del vino de Montilla-Moriles, ya en su $33^{\circ}$ edición, donde participan más de 20 bodegas y a la que acuden unas 80.000 personas (Galvez el al, 2015). Degustar, observar y conocer los vinos de la tierra es una forma de promocionar la región en cuanto al turismo. Como singular festejo podemos destacar la Fiesta del Vino y de la Tapa que viene celebrándose desde hace doce años como complemento a la Fiesta de la Vendimia Montilla-Moriles. Otro ejemplo es La Cata Flamenca de Montilla celebrada anualmente y que va por su $43^{\circ}$ edición posee una larga tradición y un gran atractivo turístico por unir dos potencialidades de la zona: el vino y el flamenco. De hecho, el certamen se celebra dentro de la Fiesta de la Vendimia Montilla-Moriles, junto a la Fiesta del Vino y la Tapa y otros concursos y actividades culturales que tienen lugar el primer fin de semana de septiembre de cada año en la localidad montillana.

Se conmemora, así, la cosecha de la uva de los pagos que conforman la prestigiosa Denominación de Origen Montilla-Moriles, y aunque los orígenes de la Fiesta de la Vendimia se remontan al siglo XIX, la Cata Flamenca comienza a celebrarse en 1970, justo cuando la fiesta es declarada De Interés Turístico Nacional.

Estos tres festivales son ejemplos de una oferta singularizada, apegada a la calidad de los productos locales, donde ambos municipios se implican y se consigue satisfacer y fidelizar al visitante desde un primer momento, además de reforzar el concepto de evento gastronómico específico de la zona.

\section{Conclusiones}

Montilla y Moriles son los dos municipios que dan nombre a la Denominación de Origen Protegida, son conocidos internacionalmente como tierra de vinos de calidad excelente.

El turismo se ha convertido en posible solución y alternativa económica de algunos de los problemas que han surgido en zonas agrarias y que los municipios de Montilla y Moriles no le son indiferentes: incorporación laboral de la mujer, altas tasas de paro, éxodo rural, dependencia del sector primario, etc.; La práctica y el crecimiento de esta actividad generará y diversificará las rentas, producirá pluriactividad, creará empleo, disminuirá el éxodo rural de jóvenes que podrán desarrollar su formación, etc. Por lo tanto, es preciso ofrecer aquel servicio o producto que se adapte a la demanda del consumidor turístico, y para ello es imprescindible ofrecer un servicio o producto que se ajuste a las necesidades reales del consumidor actual, por ello es necesario conocer su perfil (Millan et al, 2011), se entiende pues que una de las líneas de investigación a seguir es el estudio de la demanda de turismo a lo largo de las bodegas que conforma la Denominación de Origen protegida, o estudiar al menos las principales bodegas receptores de las visitas, así como un estudio individualizado de cada uno de los lugares de interés ofertados dependiendo de cada tipo de turista. Hemos de concluir, a modo general, que Montilla y Moriles como municipios presentan unas expectativas positivas de desarrollo turístico. Las principales conclusiones podemos definirlas:

$\checkmark$ Del estudio de campo realizado, vía bibliografía y vía entrevistas con el sector, se desprende el potencial turístico que presentan ambos municipios. Tenemos ejemplos del elevado número de visitas a determinadas bodegas en Jerez, o diferentes Denominaciones de Origen. 
$\checkmark$ Se pueden vertebrar y unir a la actual ruta del vino con motivaciones hacía el turismo natural, patrimonial, religioso o gastronómico visitando y enseñando diferentes lugares dado su atractivo, usando las propias visitas a las bodegas como propio atractivo y singular para el turista, pero enlazando con otras motivaciones.

$\checkmark$ Como se ha resaltado con respecto al número de turistas que visitan determinadas Bodegas no están identificadas el número de entradas o visitas en un sentido global ni por segmentos o tipologías turísticas siendo esta una vía de investigación de futuro. Tampoco existe un organismo provincial específico que aglutine dichas estadísticas, con el fin de medir tanto la oferta como la demanda y poder poner a disposición del empresariado, administraciones públicas, cooperativas, bodegas y otros organismos una herramienta de gestión útil.

$\checkmark$ Existe una rica gastronomía en la zona basada en la singularidad de los productos, entre ellos los productos unidos a la Denominación de Origen sin embargo no aparecen restaurantes o establecimientos con menciones en guías de prestigio.

$\checkmark$ No podemos afirmar que existe una clara insuficiencia para cubrir la demanda actual en cuanto al número de establecimientos de hospedaje y de hostelería, dado que, al no tener el dato del número de visitantes o excursionista en cuanto a capacidad, no podemos realizar un contraste entre la oferta y demanda turística. El ubicar un punto testigo para el observatorio de turismo de la provincia es considerado la solución a esta falta de información (Hernández, 2016b).

$\checkmark$ La gastronomía es un elemento diferenciador de la zona, el hecho de que prácticamente el $50 \%$ de los establecimientos hosteleros presenten una oferta propia y singular de la zona así lo demuestran.

$\checkmark$ Existen en la zona suficientes festivales consolidados para que sirvan de plataforma de comercialización del turismo en los municipios.

$\checkmark$ Como alternativa a la promoción de ambos municipios y de la zona una opción a tener en cuenta es unir el mismo con las distintas celebraciones o fiestas de los dos municipios y la zona. El turismo de festivales, gastronómico o el de naturaleza pueden ser motivaciones importantes para incrementar el número de visitantes a la zona.

$\checkmark$ Se deberían potenciar diferentes actividades de ecoturismo dentro del de la propia ruta, ya que el principal usuario del mismo es el turista joven, lo cual generaría más ingresos en los municipios y se obtendría, por tanto, un mayor desarrollo económico. Existen determinadas Bodegas que han recibido premios y menciones en cuanto al desarrollo de producto ecológico lo cual le da un valor añadido a la zona que ambos municipios deberían aprovechar.

$\checkmark$ El turismo de congresos pese a ser potencialmente factible actualmente no existe una estructura de hoteles y restaurantes, si bien como alternativa se presentan alojamientos de otros municipios incluida la capital.

Por último, también hemos realizado unas breves referencias a la necesidad de articular y potenciar diferentes tipos de turismo en torno a la figura de la Denominación de Origen y la ruta del vino ya creada y consolidada, dado que las estructuras están ya ahí y sería relativamente fácil ponerlas en valor y promocionándolas al exterior. Esto conllevaría un crecimiento de la oferta de restaurantes y de hospedaje como consecuencia de incremento de flujos turísticos. 


\section{Bibliografía}

Armesto, X. A. y B. Gómez (2004): "Productos agroalimentarios de calidad, turismo y desarrollo local: el caso del Priorat”, Cuadernos Geográficos, 34, pp. 83-94.

Barrera, E. y Bringas, O. (2008) Las Rutas Alimentarias: Una arquitectura turística basada en la identidad de los alimentos Scienze Gastronomiche - Università degli studi di Scienze Gastronomiche N 8 - Mayo 2008.

Berveland, M. (1988). Wine tourism in New Zealand - Maybe the industry has got it right. International Journal of Wine Marketing, v. 10 (2), 24-33.

Bruwer, J. (2003). South Africa wine routes: Some perspectives on the wine tourism industry's structural dimensions and wine tourism product”. Tourism Management, v. 24, 423-435.

Cambourne, B. y Macionis, N. (Eds.), Wine tourism around the world: Development, managements and markets, 102-114. Oxford: Butterworth-Heinemann.

Castillejo, J. M., \& del Castillo García, F. (1994). Influencia del clima en el viñedo de la denominación de origen Montilla-Moriles. In Actas del VII Coloquio de Geografía Rural:(comunicaciones) (pp. 166-171). Servicio de Publicaciones.

Denominación de Origen Protegida Montilla-Moriles http://www.montillamoriles.es/

Demhardt, I. (2003). Wine and tourism at the Fairest Cape: Post-apartheid trends in the Western Cape Province and Stellenbosch (South Africa). Journal of Travel and Tourism Marketing, v. 14 (3), 113-130.

Domingos, S. y Henriques, C.(2015): Património Gastronómico E Sua Valorização PelasEntidades Público-Privadas - O Caso Da Doçaria Da Região Do Algarve. International Journal of Scientif Management and Tourism, 3, 213-232.

Dowling, R. y Carlsen, J. (Eds.) (1999). Wine tourism: perfect partners. Proceedings of the First Australian Wine Tourism Conference. Canberra: Bureau of Tourism Research.

Eisenhardt, K. M. (1989). Agency theory: An assessment and review. Academy of management review, 14(1), 57-74.

Fuentes García, F. J., y Veroz Herradón, R. (2000). Plan estratégico de la denominación de origen Montilla-Moriles. Servicio de Publicaciones de la Universidad de Córdoba, Córdoba.

Frochot, I. (2000). “Wine tourism in France: a paradox?”. En: Hall, C.M.; Sharples, L.; Cambourne, B. y Macionis, N. (Eds.), Wine tourism around the world: Development, managements and markets, 67-80.

Gálvez, J. C. P., Fernández, G. A. M., \& Guzmán, T. L. G. (2015). Motivación y satisfacción turística en los festivales del vino: XXXI ed. cata del vino MontillaMoriles, España. Tourism \& Management Studies, 11(2), 7-13.

Getz, D. y G. Brown (2006): “Critical Success Factors for Wine Tourism Regions: A Demand Analysis”, Tourism Management, 27, pp. 146-158.

Gobbi, J. (2003). 24 Turismo y autencidad: hacia una propuesta relacional para el estudio de la interacción entre nativos y turistas en las comunidades locales. Texto apresentação no Congresso Virtual de Turismo. 
Gómez, A. C., y de Haro Giménez, T. (1990). Actuación comercial de las cooperativas vitivinícolas: análisis crítico del caso Montilla-Moriles. Revista de Estudios Agrosociales, (151), 157-190.

Hall, C. M. y R. Mitchell (2000): "Wine Tourism in the Mediterranean: A Tool for Reestructuring and Development", Thunderbird International Business Review, vol. 42 (4), pp. 445-465.

Hall, C. M., Johnson, G., Cambourne, B., Macionis, N., Mitchell, R., \& Sharples, L. (2000). Wine tourism: an introduction. Wine tourism around the world: Development, management and markets, 1-23.

Hashimoto, A., \& Telfer, D. J. (2003). Positioning an emerging wine route in the Niagara region: Understanding the wine tourism market and its implications for marketing. Journal of Travel \& Tourism Marketing, 14 (3-4), 61-76.

Hernández Rojas, R. Millán Vázquez De la Torre, M.G (2015). Análisis de la oferta en restauración de la ciudad de Córdoba y su implicación con las rutas gastronómicas de la provincia. Revista de turismo y desarrollo local, 8 (18), 1-13.

Hernández Rojas, R. Millán Vazquez De la Torre, M.G. y Dancausa Millan G. (2016a). Tourism Observatory: a need for management and gastronomy of Cordoba province. International Journal of Scientific Management and Tourism, 2(4), 69-80.

Hernández Rojas, R. Millán Vazquez De la Torre, M.G y Dancausa Millan G. (2016b). Análisis del turismo gastronómico en Córdoba y Provincia: Estrategias de gestión. Cordoba, Excma. Diputación de Córdoba.

López-Guzmán Guzmán, T. J., Lara de Vicente, F. y Merinero Rodríguez, R. (2007). Las rutas turísticas como motor de desarrollo económico local. La ruta del Tempranillo. Estudios Turísticos, $\mathrm{n}^{\circ}$ 167, 131-145.

López-Guzmán, T. J., \& Millán-Vázquez de la Torre, G. (2008). Análisis econométrico del enoturismo en España Un estudio de caso. Estudios y perspectivas en turismo 17. 2, 98-114.

López-Guzmán, T. J. L. G., Cañizares, S. M. S. (2008). La creación de productos turísticos utilizando rutas enológicas. Special Issue-Número Especial Turismo grastronómico y enoturismo Gastronomic and wine tourisme, 6, 159.

López-Guzmán, T., Cañero Morales, P. M., Moral Cuadra, S., Orgaz-Agüera, F. (2016). An exploratory study of olive tourism consumers. Tourism and Hospitality Management, 22(1), 57-68.

Luque-Vílchez, M. (2015). La necesidad de internacionalización: entrada de la Denominación de Origen Montilla-Moriles en los mercados BRIC. Cuadernos de Estudios Empresariales, 25, 11.

MacCannel, D. (1976). The tourist. A new Theory of the Leisure Class.

Medina, M., López, P. A., \& Moreno, J. J. (1988). Caracterización del envero y madurez en la uva de vitis vinífera" Pedro Ximenez" cultivada en la zona MontillaMoriles. Revista de agroquímica y tecnología de alimentos, 28(2), 274-284.

Millán Vázquez de la Torre, M. G., y Navarro, A. M. (2008). Rutas turísticas enológicas y desarrollo rural. El caso estudio de la Denominación de Origen Montilla-Moriles en la provincia de Córdoba. Papeles de geografía, (47-48), 159-170. 
Millán Vázquez de la Torre, G., Hernández Rojas, R., y Navajas Romero, V. (2016). The study of gastronomic tourism in Cordoba and the association of the cuisine. An econometric analysis. Tourism and Hospitality Management, 22(2), 173-191.

Millán Vázquez de la Torre, M. G., Morales Fernández, E. J., \& Agudo Gutiérrez, E. M. (2010). El oleoturismo como motor de desarrollo rural: La denominación de origen de montoro-adamuz. Mundo agrario, 11(21).

Millán Vázquez de la Torre, M G. (2011): Las empresas alimentarias nuevo motor del turismo industrial en la provincia de Córdoba. Análisis del perfil del turista. Rotur/Revista de ocio y turismo, 4, 89-116.

Millán Vázquez de la Torre, M G., y Morales-Fernández, E. J. (2015). Denominaciones de origen protegidas (DOP) y turismo gastronómico: una relación simbiótica en Andalucía. Gran tour , revista de investigaciones turíticas, (6).

Mitchell, R. y Hall, C.M. (2003). Seasonality in New Zealand winery visitation: An issue of demand and supply. Journal of Travel an Tourism Marketing, v.14 (3/4), 155173.

Morales, E. F. y Gutiérrez, E. M. A. (2010). El oleoturismo como motor de desarrollo rural: La denominación de origen de montoro-adamuz. Mundo Agrario: Revista de estudios rurales, 11(21), 14-27.

Morillas Javier (2001). Turismo: empresa y comunicación. Cámara de Comercio de Madrid en colaboración con la Universidad San Pablo-CEU Madrid, 2001, 328 páginas

Muñoz-Fernández, G. A., Gálvez, J. C. P., \& Guzmán, T. L. G. (2016). Synergy between wine, travel and enogastronomic festivals. An analysis of motivation and satisfaction. International Journal of Scientific Management and Tourism, 2(3), 225240.

Preston-White, R. (2000). Wine routes in South Africa: En Hall, C.M.; Sharples, L.;

Romero Moreno, D. (2008): “Análisis del sector de la restauración en España: oferta y peso económico actual”. Innovación y Experiencias Educativas, (13).

Ruta del vino Montilla- Moriles. http://www.turismoyvino.es 03/01/17

Stephen C.,(1990): The search for authenticity: review essay of Dean MacCannell, the touris "Berkeley Journal of Sociology: a critical review” n 35,1990,151-156.

Sharples, Liz (2002): Wine Tourism in Chile... A Brave New Step for a Brave New World”, International Journal of Wine Marketing, nº 14, 2 pp. 43-53.

Shudson M., Review essay: on tourism and modern culture, en "American Journal of Sociology, $\mathrm{n}^{\circ}$ 84,1979, pp 1249-1258

Skinner, A. (2000). Napa Valley, California: A model of wine region development. En: Hall, C.M.; Sharples, L.; Cambourne, B. y Macionis, N. (Eds.), Wine tourism around the world: Development, managements and markets, 283-296. Oxford: ButterworthHeinemann.

Szivas, E. (1999). The development of wine tourism in Hungary. International Journal of Wine Marketing, 11-2, 7-17.

Telfer, D. J. (2001). Strategic alliances along the Niagara wine route. Tourism Management, v. 22, 21-30.

Thevenin, C. (1996). Quands le vignerons font du tourisme. Espaces, v. 140, 43-48. 
Titos Moreno, A. , y de Haro Giménez, T. (1990). Incidencia de las reglamentaciones española y comunitaria en los vinos de la zona" Montilla-Moriles". Revista de Estudios Agrosociales, (151), 139-156.

Urry J (1990):The tourist gaze, Leiusure and travel in contemporary societies, Sage, London.

Williams, P. W. y Kelly, J. (2001). Cultural wine tourists: Product development considerations for British Columbia's resident wine tourism market. International Journal of Wine Marketing, v. 13 (3), 59-76.

Williams, P. y Dossa, K. (2003). Non-resident wine tourist markets: implications for British Columbias emerging wine tourism industry. Journal of Travel and Tourism Marketing, v.14 (3/4), 1-34. 